Vol. 7I, N. ${ }^{\circ}$ II3 (mayo 20I9), I5-27

\title{
UNA LECTURA FEMINISTA DEL PENSAMIENTO ANDROCÉNTRICO DE LA ECONOMÍA Y LA SOSTENIBILIDAD DE LA VIDA: EL CASO DE LAS MUJERES DEL BARRIO MARÍA AUGUSTA URRUTIA
}

\author{
XIMENA CABRERA MONTÚFAR \\ Universidad Andina Simón Bolívar
}

Recepción de manuscrito: 26 de abril de 2019

Aceptación de versión final: 30 de abril de 2019

\begin{abstract}
RESUMEN Este artículo propone un análisis feminista sobre la invisibilización del trabajo doméstico y de cuidados de gran parte de las mujeres que asumen las jefaturas del hogar. De tal forma se revisó y se organizó un marco teórico sobre las teorías feministas, las cuales cuestionan al patriarcado como un sistema cultural imperante y la teoría feminista económica que logra desmontar el androcentrismo acuñado al sistema económico.
\end{abstract}

palabras ClaVe Economía, cuidado, reproducción, producción, tiempo.

ABTRACT This article proposes a feminist analysis on the invisibilization of domestic work and the care of a large part of the women who assume the head of the household. In this way, a theoretical framework on feminist theories was reviewed and organized, which question patriarchy as a prevailing cultural system and the economic feminist theory that manages to dismantle androcentrism coined in the economic system.

KEYWORDS Economy, care, reproduction, production, time.

JEL CODES B54, J16, J17.

\section{INTRODUCCIÓN}

El presente artículo de investigación ensaya de manera crítica cómo la economía androcéntrica clásica y neoclásica de la economía - vigente hasta la actualidad- está imbricada de valores culturales y morales patriarcales, los cuales reproducen la retórica de la domesticación femenina, la servidumbre y el cautiverio de las mujeres en el trabajo doméstico y de cuidados en el hogar. Desarrolla a partir de un locus teórico feminista un estudio de caso sobre la invisibilización de la incidencia económica de las mujeres del barrio María Augusta Urrutia de la ciudad de Quito. ${ }^{2}$ Este estudio de caso se lo realizó a partir de la metodología de Investigación Acción Participativa, la cual posee un componente cualitativo y cuantitativo. 
Las mujeres del barrio trabajan en el cuidado de sus familias y de su comunidad sin recibir ningún tipo de remuneración económica, el uso del tiempo en este contexto es invertido en gran medida en actividades de cuidados, por lo que la organización de las horas en las cuales ejecutan dichas actividades tiene una referencia y comprensión distinta del uso del tiempo en relación a un trabajo asalariado, el cual está ordenado por las dinámicas del mercado, por lo que este estudio de caso distingue como el uso del tiempo es funcional a la sostenibilidad de la vida, a lograr el bienestar y la existencia de los/las más cercanas, asimismo este artículo busca evidenciar cómo este trabajo no remunerado de las mujeres incide en la economía familiar y nacional, por lo que se pone en práctica un método de cuantificación y valoración económica de este trabajo.

\section{EL ANDROCENTRISMO DEL PENSAMIENTO ECONÓMICO: EL HOMO ECONOMICUS Y LA RETÓRICA DE LA DOMESTICIDAD FEMENINA}

Históricamente, los estudios clásicos sobre economía han asignado un papel central a los hombres, sus principales postulados poseen una carga y un sesgo patriarcal indiscutible. La ciencia económica tradicional y clásica ha sido desarrollada de manera ahistórica al género (desconoce la inequidad), excluye a las mujeres que han contribuido a lo largo de la historia en la conformación de los sistemas económicos y en los recursos monetarios de las sociedades; Cristina Carrasco, en su obra Mujeres y economía, señala en este sentido que:

Los pensadores clásicos — estudiosos de lo que ha venido a llamarse economía política - viven un período de transición y restructuración ligada naturalmente al proceso de industrialización. La producción orientada al mercado se está separando de la producción doméstica destinada al autoconsumo familiar. Esta situación colabora en que sus análisis se centren en la producción capitalista y su instrumental analítico y conceptual tome como referencia exclusivamente este tipo de producción. Así, comienza una tradición que ignora la división por sexo del trabajo y oculta el trabajo familiar doméstico y su articulación con la reproducción del sistema capitalista. (2003, pág. 16)

Como analiza la autora, los estudios económicos clásicos se centraron por un largo tiempo tan solo en la esfera de la producción, dejando de lado todo el trabajo reproductivo familiar que realizaban las mujeres, por lo tanto, invisibilizando el trabajo de cuidado aun en aquellos casos en el que las mujeres también laboraban en los espacios productivos, por ejemplo, al hablar de las mujeres trabajadoras de los siglos XVIII, XIX y xx los estudios históricos y económicos no ahondaron en las formas en cómo estas realizaban jornadas extendidas no solo en sus puestos de trabajo, sino también con el trabajo en sus hogares ejecutando actividades de cuidado; así, estos estudios económicos dejan de lado el aporte que las mujeres otorgaban a la economía a partir del espacio privado.

El trabajo que realizan las mujeres está negado en los estudios de los pensadores clásicos, valoraron las actividades de cuidado en tanto obligación femenina, debido a los roles de género impuestos históricamente por el patriarcado, por lo tanto, no se ubicaron a las actividades de cuidados en la esfera de lo económico (England, 1993). Los autores clásicos, entre ellos 
los principales pensadores a quienes nos referimos, Adam Smith, David Ricardo, no necesitaron medir en términos monetarios los efectos de las actividades de cuidado dentro de las economías locales y nacionales, pues consideraban que éstas no generaban valor, como Smith lo señala: «[E]ste tipo de trabajo no debe entrar en conflicto con la teoría del valor, puesto que, al no formar parte de la producción e intercambio de mercancías, queda fuera de su campo de aplicación» (Smith, 1976, pág. 299).

Como reflexiona M. Ferber en su crítica a los estudios de la economía intitulada Más allá del hombre económico, el pensamiento económico clásico y neoclásico (la escuela marginalista) poseían en sus argumentos una carga bastante moral adscrita sobre todo a valores patriarcales que reproducen los estereotipos y roles de género, explora la desconexión de esta teoría económica con la realidad social, sino con un individualismo masculino que interpreta los fenómenos económicos a su conveniencia (Ferber y Nelson, 2004, pág. 14). El espacio familiar fue interpretado como un espacio pacífico y tranquilo bajo el supuesto de que no existen conflictos de clase, ni económicos y, si los hubiera, se dio por contado que las mujeres poseen estrategias mediadoras para resolver dichos conflictos a través de la sensibilidad y la afectividad. Las disputas en este sentido son entendidas como válidas solo en el mercado y se desarrollarían solo en el espacio donde se generan ganancias. Este postulado ha sido validado tanto por la tradición neoclásica y la teoría marxista (Carrasco, 2003).

La teoría económica androcéntrica que argumenta los fenómenos económicos desde la visión masculina y en la que el protagonista de dichos fenómenos siempre son los hombres, está definitivamente interconectada ideológicamente con el proyecto patriarcal histórico del que habla Lerner. ${ }^{3}$ La teoría feminista, en este sentido, invita a considerar que los sistemas económicos y el actual sistema económico capitalista no es más que la materialización de los postulados androcéntricos. Así, hasta la teoría marxista, la cual aporta de manera importante al estudio crítico y político de la economía (Karl Marx y Friedrich Engels), evidenció que el espacio de la familia era un espacio reproductivo no generador de valor.

Retomando la obra de Karl Marx, El capital, libro primero, se puede notar de manera clara cómo el autor analiza de manera desacertada la cuestión de la reproducción de la fuerza de trabajo de los obreros, tratándolo como parte de su instinto de conservación: «La constante conservación y reproducción de la clase obrera es siempre condición constante de la reproducción del capital. [Pero] el capitalista puede confiar tranquilamente su cumplimiento al instinto de conservación y reproducción de los trabajadores» (Marx, 1867, pág. 572). Según estos términos utilizados por Marx, los trabajadores generan por sí mismos su bienestar, su conservación, inobservando el trabajo doméstico y de cuidados que las mujeres ejercían en los hogares y que continúa siendo una constante en la actualidad. Marx, al referirse a las funciones familiares, no logra intuir que son las mujeres, en el contexto histórico de los inicios de la industrialización, quienes lograron acceder al trabajo en la fábrica, no poseían las mismas condiciones que los trabajadores hombres; mujeres que debían realizar dobles jornadas de trabajo, deberían pagar los servicios de reproducción de su hogar cuando ellas no pudieran suplirlo, como si éstas tuvieran la posibilidad económica de siempre hacerlo por un lado, desconociendo las responsabilidades de los hombres en los hogares, lo que hace notar que en sus líneas argumentativas la responsabilidad de la familia es inminentemente de las mujeres (Marx, 1867, pág. 395). 
Este breve marco analítico logra reflejar el criterio androcéntrico del pensamiento económico clásico, por lo que la economía feminista ha ido desarrollando posturas críticas hacia esta manera masculinizada de concebir la economía.

\section{LA ECONOMÍA FEMINISTA Y SUS APORTES AL CUESTIONAMIENTO DE LA SERVIDUMBRE DOMÉSTICA}

El hecho de que, de manera poco inocente, la teoría económica de los pensadores clásicos y neoclásicos no cuestionaran las relaciones de género en la economía y los conflictos relacionados a la división sexual del trabajo, incitó a que las teóricas feministas organicen una serie de teorías que cuestionan la exclusión de las mujeres del sistema económico.

La división del trabajo en la familia fue una de las primeras preocupaciones de las feministas, quienes, enfocadas en la ideología marxista del sistema de opresión, encuentran que en su base (ideología marxista) se encuentran los hombres (Carrasco, 2003, pág. 21), lo cual implicó profundizar el análisis teórico de las opresiones más allá de la explotación a los trabajadores hombres y la lucha de clases, hacia la división sexual del trabajo y las relaciones de opresión de género en la economía. El concepto de división sexual del trabajo explica la asignación generalizada de tareas específicas en el trabajo a hombres y mujeres, en el que subvalora el trabajo de las mujeres, pero además da cuenta de la doble presencia de las mujeres en la esfera productiva y la esfera denomina reproductiva, que conlleva a una subestimación de la reproducción en relación a la producción; además, como señala Cristina Borderías, la economía feminista logra dimensionar la importancia del trabajo reproductivo para que el productivo exista, un trabajo invisible para que la producción sea visible (Borderías, 1999).

El análisis para las economistas feministas se desarrollaría en estas dimensiones sociales, en las cuales la construcción del género desemboca en la opresión, subordinación y exclusión de las mujeres del sistema económico. Autoras como Aleksandra Kolontái en los años 70, economistas y estudiosas como Lourdes Benería en los 80, Marianne Ferber, Julie Nelson, Nancy Folbre en los 9o, Cristina Carrasco, Corina Rodríguez, Amaia Pérez Orozco, Mercedes d'Alessandro en los 2000, entre muchas otras autoras actuales y contemporáneas, reflexionan de manera rigurosa en sus obras cómo la economía tradicional ha reproducido por siglos la desigualdad de género en la economía. Estos estudios empezaron a cuestionar la posición domesticada de la mujer en el hogar y la carga de trabajo doméstico que imposibilitaba a las mujeres a ser partícipes y ubicarse en otro espacio que no sea el privado del hogar; lo que les imposibilitaba entonces desempeñarse en otros ámbitos como el profesional o continuar con sus proyectos de vida (Benería, 1984).

La teoría económica feminista contribuye con un salto epistemológico desde la forma en cómo se investiga y se analiza a las relaciones de poder atravesadas por el género en la economía. A partir de una lectura crítica al pensamiento androcéntrico de la economía, tomando en cuenta en cierta medida cuestiones como las relaciones de opresión trabajadas por el marxismo, se construye de manera sistemática un corpus teórico cuestiona la posición desigual en la que las mujeres se desarrollan dentro del sistema económico. Explica cómo funcionan las relaciones de poder en la familia, al igual como sucede en el mercado. Las estudiosas postulan 
que las actividades subjetivas, las actividades de afecto, de cuidado y las labores domésticas eran el centro fundamental de la economía, como señala Silvia Federici:

[...] [E]l capital ha tenido mucho éxito escondiendo nuestro trabajo. Ha creado una obra maestra a expensas de las mujeres. Mediante la denegación del salario para el trabajo doméstico y su transformación en un acto de amor, el capital ha atado dos pájaros de un tiro. Primero, ha obtenido una cantidad increíble de trabajo casi gratuito [...]. (2013, pág. 38)

En este marco de investigación económica feminista, las teóricas logran ubicar un exégesis de la procedencia de la omisión de las mujeres en la economía, y tal como se lee en Calibán y la bruja de Silvia Federici, el patriarcado asignó a las mujeres la categoría precapitalista, es decir, el sistema económico se transformó y devino en capitalista, pero las mujeres, sobre todo quienes trabajan en el hogar, siguieron siendo tratadas como sujetos precapitalistas que no inciden en la economía (Federici, 2010); lo que reflexiona Federici es el Estado precapitalista — de servidumbre, domesticación y no producción de mercancías de las mujeres que trabajan en actividades domésticas en el hogar- en tanto no son productoras de valor y sus actividades no se encuentran en el mercado de trabajo asalariado.

El feminismo económico contribuye a pensar en que el capitalismo no solo funciona a partir de la explotación obrera, sino que en su centro se encuentra todo el trabajo no pagado a las mujeres, trabajo doméstico y de cuidados, cuyas actividades posibilitan que la fuerza de trabajo tenga las posibilidades básicas para desarrollarse en el mercado. Como lo estudió el feminismo radical estadounidense a partir de autoras como Millet, el patriarcado no debe ser entendido como un modelo de subordinación reducido a una sola dimensión social en las mujeres, sino que plantea que es a través de la opresión sexual y del cautiverio de la reproducción impuesto por el patriarcado (Millet, 2010), que generan políticas ideológicas y económicas dentro de un proyecto cultural interrelacionado y constitutivo del capitalismo patriarcal.

En el sistema capitalista, los cuerpos son reconfigurados e impuestos como una corporalidad solo para el trabajo; como una máquina productora de valor en el sistema capitalista. En este sentido, las mujeres han sido ubicadas como una máquina corporal biologizada, útil solo para la reproducción (Federici, 2010). Las mujeres valen en tanto paren o reproducen, automáticamente la cuestión de los cuidados y las actividades domésticas son asignadas naturalmente como características inherentes al «ser reproductivo», la abnegación y el altruismo en las mujeres se inculcan culturalmente de una manera habitual.

El trabajo como categoría en este sentido feminista es resignificado, transgrede el sentido de trabajo que otorgó la economía clásica y la teoría marxista, el trabajo para la economía feminista, más allá de si es asalariado o no, pues no es el salario lo que dispone qué es trabajo y qué no lo es, trabajo son todas las actividades que realizan las mujeres en los hogares en términos de que posibilitan la existencia de fuerza de trabajo y, más allá de esto, también es trabajo porque no son actividades que les corresponde realizar a las mujeres por su naturaleza femenina. El trabajo debe ser determinado como una función acogida por las personas para poder sobrevivir en un sistema económico en el que el consumo y la sobrevivencia es su principal principio. Releyendo la historia y ubicándose en la línea feminista económica, se comprende que el ser amas de casa no es una labor que las mujeres cumplen porque está en su 
naturaleza, sino que esta socialización de la servidumbre doméstica de las mujeres ha durado siglos de socialización del patriarcado.

El trabajo doméstico es invisible sobre todo porque no está retribuido y porque ningún pensador económico ha querido verlo, la mujer era el sujeto a la cual el capitalismo había endosado el trabajo doméstico, condicionándole así toda su vida. Por lo tanto, quedan expuestos dos polos contrarios el de la producción: la fábrica y la reproducción: actividades del hogar; no obstante, el contexto social no poseía relaciones sociales libres, sino que se adscribía al funcionamiento de la fábrica, en esta fábrica social (cultural) no solo estaba el denominado obrero social, sino que toda ello giraba en torno a un segundo polo: el hogar, cuyo sujeto era la mujer (Costa, 2006). Como se observa, el trabajo teórico feminista, que realiza una crítica a la economía androcéntrica del Homo economicus, centró su atención sobre todo en la desigualdad de género en la esfera económica.

En términos de que este breve análisis teórico posea un sentido práctico, a continuación se detalla de manera resumida, la aplicación de una metodológica de valoración económica desde la teoría feminista, al estudio de caso específico sobre el trabajo de cuidados no remunerado de las mujeres del barrio María Augusta Urrutia.

\section{EL TRABAJO DE CUIDADOS DE LAS MUJERES DEL BARRIO MARÍA AUGUSTA URRUTIA: TIEMPO NO ESCINDIDO Y SU INCIDENCIA ECONÓMICA}

Las mujeres del barrio María Augusta Urrutia son mujeres que realizan trabajo de cuidados no remunerados en sus hogares, ellas se han identificado como madres jefas de hogar ${ }^{4}$ en términos de que son las únicas, y de manera abnegada, que sostienen a sus familias, satisfacen las necesidades de éstas y de su comunidad. Son mujeres que aportan a la economía de la ciudad y sostienen la vida de los y las trabajadoras de su barrio, a continuación se detalla de manera resumida cómo estas mujeres inciden en la economía.

Las mujeres jefas de hogar del barrio realizan las actividades domésticas de diversas formas. Puede haber actividades que se las realiza en tiempos sincronizados y en un proceso de continuidad, es decir, que algunas actividades se las realiza en relación a un proceso determinado de la vida de los/las familiares, esposos y convivientes. Son actividades que deben realizarse en un espacio y tiempo continuo, un tiempo relacionado a las necesidades de la vida de los miembros del hogar, sigue una orientación en función de la alimentación, la limpieza, el cuidado de hijos, parientes con discapacidad, sostenimiento emocional y afectivo, entre otras cosas (ver Tabla 1).

El tiempo discontinuo, por otro lado, está caracterizado entonces por la interpretación de que el tiempo se fragmenta. Esta división se la puede observar cuando las actividades, sean éstas para el cuidado de otros familiares o de tareas domésticas, se tengan que realizar fuera de casa, en otros espacios en los que la madre jefa de hogar no tiene el control. El espacio externo es un lugar en el que rigen otras normas, reglas y hasta otro tiempo: el tiempo de entrada de los niños a la escuela, el tiempo en el que abren las oficinas para pagar los servicios básicos, el tiempo en el que abren y cierran las tiendas o supermercados, entre otras cosas. La discontinuidad, entonces, se refiere a que se rompe con la sincronía de las actividades dentro del espacio homogéneo, si se quiere, del hogar (ver Tabla 2). 
Tabla 1: Tiempo continuo de trabajo no remunerado

\begin{tabular}{|c|c|c|}
\hline Tiempo continuo & Actividades domésticas & $\begin{array}{l}\text { Actividades de cuidado: } \\
\text { (bebés, niños/as, personas } \\
\text { con alguna discapacidad, } \\
\text { convivientes o esposos) }\end{array}$ \\
\hline \multirow{10}{*}{$\begin{array}{l}\text { Tiempo sincronizado, no } \\
\text { fracturado, es un tiempo en el que } \\
\text { se realizan actividades de manera } \\
\text { jerárquica, organizada y en un } \\
\text { espacio determinado: el hogar, La } \\
\text { comunidad de este tiempo es } \\
\text { posible porque se las realiza al } \\
\text { interno de la vivienda. }\end{array}$} & Preparación del desayuno & Preparación del biberones, papillas \\
\hline & Preparación del almuerzo & $\begin{array}{l}\text { Cambio de ropa a niños(as y bebés, } \\
\text { personas con discapacidad }\end{array}$ \\
\hline & $\begin{array}{l}\text { Preparar mesa y entregar } \\
\text { el desayuno a familiares, } \\
\text { esposo o conviviente de } \\
\text { ser el caso }\end{array}$ & $\begin{array}{l}\text { Cambiar pañales a bebés y familiares } \\
\text { con discapacidad }\end{array}$ \\
\hline & Bañar y vestir niños & Hacerle dormir al o la bebé/es \\
\hline & Lavar vajilla & $\begin{array}{l}\text { Ayudar en tareas escolares a } \\
\text { niños/as en casa }\end{array}$ \\
\hline & Arreglo y limpieza de casa & $\begin{array}{l}\text { Arreglar y limpiar cosas, } \\
\text { herramientas de trabajo del } \\
\text { esposo o conviviente }\end{array}$ \\
\hline & $\begin{array}{l}\text { Alimentación a mascotas } \\
\text { y a especies menores }\end{array}$ & $\begin{array}{l}\text { Generar satisfacción al esposo } \\
\text { o conviviente }\end{array}$ \\
\hline & Preparación de merienda & Otorgar medicamentos a familiar \\
\hline & Planchar ropa & con discapacidad \\
\hline & $\begin{array}{l}\text { Reparación de desperfectos } \\
\text { en una casa }\end{array}$ & $\begin{array}{l}\text { Dar de comer al familiar con } \\
\text { discapacidad }\end{array}$ \\
\hline
\end{tabular}

Fuente: Investigación Acción Participativa Barrio María Augusta Urrutia. Elaboración: propia.

Tabla 2: Tiempo discontinuo de trabajo no remunerado

\begin{tabular}{lll}
\hline Tiempo discontinuo & Actividades domésticas & $\begin{array}{c}\text { Actividades de cuidado: } \\
\text { (bebés, niños/as, personas } \\
\text { con alguna discapacidad, } \\
\text { convivientes o esposos) }\end{array}$ \\
\hline $\begin{array}{l}\text { Tiempo discontinuo, fracturado, la relación } \\
\text { con el espacio exterior provoca una ruptura, }\end{array}$ & $\begin{array}{l}\text { Hacer compras en tienda, } \\
\text { mercado o supermercado }\end{array}$ & $\begin{array}{l}\text { Llevar al pediatra a bebés o niños/as } \\
\text { familiares. }\end{array}$ \\
\cline { 2 - 3 } $\begin{array}{l}\text { las actividades se realizan en un tiempo que } \\
\text { instituciones, lógicas del mercado, del } \\
\text { exterior al hogar, ya no está en el control de } \\
\text { las mujeres jefas de hogar. }\end{array}$ & Pagar servicios básicos \\
$\begin{array}{l}\text { Este tiempo sediscontinúa, entre el ir y venir } \\
\text { el tiempo pasa y se desorganiza cualquier } \\
\text { planificación del tiempo del hogar. }\end{array}$ & & $\begin{array}{l}\text { Llevar almuerzos a esposo o } \\
\text { conviviente a su lugar de trabajo }\end{array}$ \\
\hline
\end{tabular}

Fuente: Investigación Acción Participativa Barrio María Augusta Urrutia. Elaboración: propia.

Además de mostrar cómo las jefas de hogar transitan en dos lógicas del tiempo y cuáles son las actividades que se realizan en cada dinámica del tiempo, es necesario mencionar que existe otro componente importante a tomar en cuenta en esta lectura. Las mujeres, a pesar de que realizan estas actividades de manera continua o discontinua, en términos del tiempo, lo más común es que ellas realicen multitareas, es decir, que realicen dos, tres, o más actividades a la vez, dependiendo de la complejidad de cada tarea. De tal manera que al tiempo se lo debe comprender como una dimensión social. El tiempo no es tiempo por sí solo, sino que es bajo las relaciones sociales. 
Es sorprendente conocer que una de las cuestiones más problemáticas de las mujeres actualmente es la pobreza de tiempo y, que en el caso, por ejemplo, del Ecuador, las mujeres a través del trabajo no remunerado doméstico invierten 1114 horas anuales de trabajo no remunerado en el hogar (representa el $20 \%$ de aporte al PIB) en relación a los hombres, con 334 horas anuales de trabajo no remunerado en el hogar (CNIG y INEC, 2018). Tiempo, energías, conocimientos de las mujeres no reconocidos ni pagados. Es notorio, entonces, que el uso del tiempo para estas mujeres está relacionado a la vida de su familia y de su comunidad porque, además, participan de otras actividades organizativas y comunitarias del barrio; el tiempo para ellas no lo distingue un salario, ni la hora de llegada, ni la hora de salida que representa la finalización de la jornada, para ellas cada actividad de cuidado finaliza cuando la necesidad ha sido satisfecha, cuando pueden probar que sus familiares continúan viviendo y poseen bienestar.

\section{METODOLOGÍA DE CASO: INCIDENCIA ECONÓMICA DEL TRABAJO DE CUIDADOS DE LAS JEFAS DE HOGAR}

Para conocer cuánto valor genera el trabajo doméstico de las mujeres madres jefas de hogar del barrio María Augusta Urrutia, es necesario cuantificar las horas que las madres jefas de hogar otorgan al bienestar de la familia y del esposo, para posteriormente calcular en relación al sueldo básico unificado (USD 366) que reciben los esposos el trabajo no remunerado por el que se sostiene dicho salario..$^{5}$ En relación a esta metodología cuantitativa retroalimentada, a partir del trabajo cualitativo de este estudio, se puede verificar que las actividades domésticas y de cuidados son un grupo de tareas primordiales para las familias del María Augusta Urrutia. En este sentido se verifica a través de los datos de la encuesta y la fórmula de cuantificación de horas en actividades domésticas, en la que se diferencian las horas de actividades de lunes a viernes y las horas invertidas en actividades los fines de semana (ver Tabla 3).

El cálculo que se describe en los cuadros anteriores evidencia que las mujeres madres jefas de hogar gastan 3 horas y 30 minutos todos los días de lunes a viernes en actividades como la preparación del desayuno, del almuerzo, de la merienda, lavado de vajilla y limpieza de cocina. Tomando en cuenta que las mujeres madres jefas de hogar realizan la mayoría de actividades domésticas en el hogar, se puede afirmar que las mujeres diariamente están liberando tiempo a sus esposos o convivientes para que ellos puedan trabajar asalariadamente. Insistiendo en que estas actividades domésticas — así como todas las actividades del hogar- deben realizarse de manera equitativa entre la madre jefa de hogar y el esposo o conviviente, ${ }^{6}$ pero teniendo en cuenta también que en la realidad las relaciones más inequitativas e injustas en esta división de tareas se generan entre las parejas, es importante analizar la relación inequitativa de género en la división de las actividades domésticas entre los esposos/convivientes y las madres jefas de hogar del María Augusta Urrutia.

\section{RESULTADO 1: TIEMPO DE ACTIVIDADES DOMÉSTICAS QUE LAS}

MUJERES DEJAN LIBRES PARA SUS ESPOSOS DE LUNES A VIERNES

Según lo que indica este ejercicio cuantitativo, los esposos o convivientes deberían realizar las actividades domésticas tal como lo hacen las mujeres jefas de hogar, es decir, que las actividades 
Tabla 3. Cuantificación del tiempo en actividades domésticas ${ }^{*}$

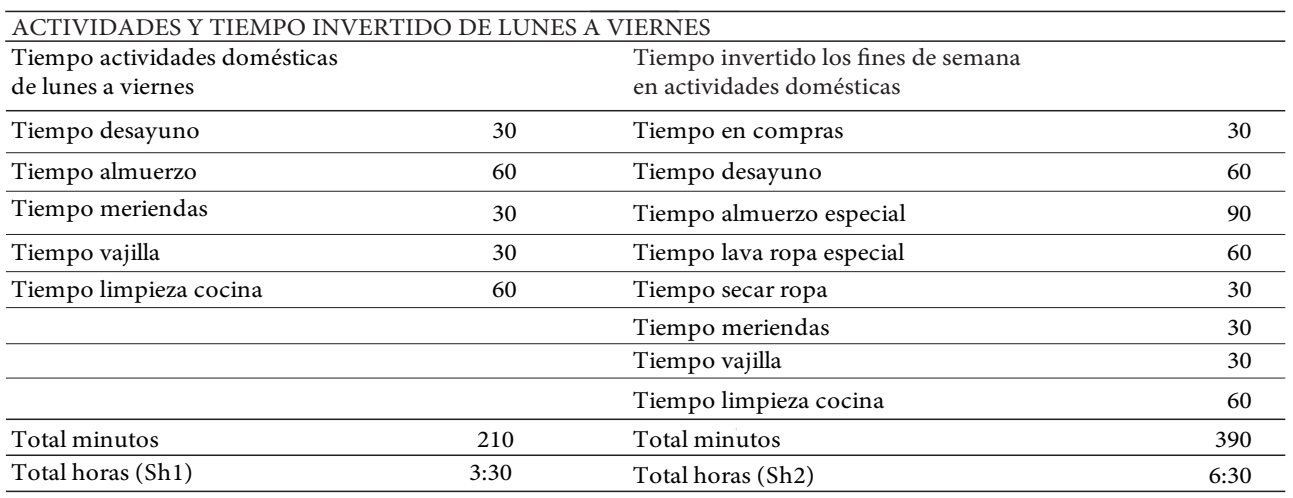

Nota: ${ }^{\star}$ Las actividades de cuidado que realizan las mujeres jefas de hogar son muchas más de las que están establecidas aquí; por el alcance de la investigación fue posible tomar en cuenta las más esenciales. Fuente: Encuesta sobre trabajo doméstico y de cuidados (MAU, 2016). Elaboración: propia.

Tabla 4. Fórmula para evidenciar el tiempo liberado a los esposos o convivientes

\begin{tabular}{ll}
\hline Fórmula & Operaciones y resultados \\
\hline$=$ Sh1 $/ 2$ (mjh-esp) & $\begin{array}{l}210 \text { minutos }(3: 30 \text { horas) } / 2 \\
\text { (personas) }=1: 15 \text { horas por } \\
\text { persona }\end{array}$ \\
\hline Variables & $\begin{array}{l}\text { (Sh1: sumatoria horas lunes a } \\
\text { viernes; mjh: madre jefa de hogar }\end{array}$ \\
& -esp: esposo o conviviente) \\
\hline
\end{tabular}

Fuente: Encuesta sobre trabajo doméstico y de cuidados (MAU, 2016). Elaboración: propia.

deberían realizarse de manera equitativa (ver Tabla 4). En este marco de expectativa y el desarrollo de la fórmula anterior, se obtiene que:

- (a) Las mujeres madres jefas de hogar gastan 3,30 horas en estas actividades domésticas de preparación de alimentos, limpieza de vajilla y cocina de lunes a viernes diariamente; mientras que los fines de semana (sábado y domingo) las actividades se extienden 6,30 horas debido al aumento de tareas que deben realizar. En relación a un enfoque de división equitativa de las tareas domésticas, ellas solo deberían invertir en estas actividades 1,15 horas entre semana y 3,15 horas los fines de semana - la mitad del tiempo gastado-, sin embargo, ellas realizan solas el total de actividades, no existe una corresponsabilidad de sus esposos/convivientes y familiares. Los roles de abnegación y altruismo se plasman en esta realidad. Las mujeres sacrifican su tiempo y energía en el bienestar de los otros.

- (b) Por otro lado, liberan 1,15 y 3,15 horas a sus esposos o convivientes de estas actividades domésticas; este tiempo al día, ellos lo utilizan (o pueden utilizarlo) para trabajar asalariadamente o por un pago, de otro modo los esposos las utilizan los fines de semana para trabajar asalariadamente en el ejercicio de horas extras o realizan actividades de recreación como jugar fútbol o ver televisión. ${ }^{7}$ La permanencia más continua de los fines de semana en el hogar genera que las jefas de hogar sientan más obligación y responsabilidad por desarrollar 
las actividades con más abnegación. Las horas que ellos utilizan en otras actividades remuneradas, o en cualquier otra actividad, son el resultado de las horas en las que las madres jefas de hogar realizan actividades domésticas. La liberación de tiempo a los esposos o convivientes es la forma más evidente en que las mujeres aportan a la economía. La fuerza de trabajo está disponible en tiempo y bienestar gracias a que las madres jefas de hogar, se encargan de realizar las actividades que también les corresponde realizar a los esposos o convivientes. Se puede afirmar que las mujeres madres jefas de hogar gastan como media $4 \mathrm{~h} 35 \mathrm{~min}$ en actividades domésticas de lunes a domingo, de las cuales a ellas solo les correspondería - bajo el enfoque de reparto equitativo de tareas - $2 \mathrm{~h} 18 \mathrm{~min}$; sin embargo, las mujeres gastan de su tiempo entre 3 h 30 min y 6 h 30 min en el transcurso de la semana. En cuanto a la liberación de tiempo que las mujeres realizan a sus esposos o convivientes, se observa que, en un cálculo de la media, serían 15 h semanales de liberación de tiempo a los esposos de trabajo doméstico y de cuidados.

Si sumamos el tiempo total que las mujeres liberan por semana para sus esposos, estaríamos hablando de 1830 minutos a la semana, es decir, 30 h 30 min en actividades domésticas esenciales. Si tenemos en cuenta que el promedio de horas laborales legales actualmente en el Ecuador se encuentra entre 40 y 48 horas semanales, estaríamos hablando que las mujeres del barrio María Augusta Urrutia trabajan entre 10 y 18 horas semanales en relación al tiempo asalariado del trabajo de sus esposos o convivientes, lo que representa un $76,25 \%$ del total de una jornada laboral; además de que hay que tomar en cuenta que muchas de estas mujeres también trabajan - de manera esporádica, no tienen trabajo estable- fuera de casa por un pago y ejercen una doble jornada de trabajo.

\section{RESULTADO 2: ¿CUÁNTO VALOR GENERA EL TRABAJO DOMÉSTICO}

DE LAS MUJERES MADRES JEFAS DE HOGAR?

Con base en la corriente de la economía feminista, en la que se consideran a las actividades domésticas como trabajo en tanto crean valor, y retomando el discurso de Federici que menciona que el salario es un fundamento importante para desnaturalizar la ideología de las actividades domésticas y de cuidado feminizadas, es necesario hacer un ejercicio de medición monetaria de este trabajo, más allá de que se considere también que el salario no es la única solución de valorización económica de las actividades domésticas. En este sentido, el salario que reciben los esposos o convivientes de las mujeres jefas de hogar del barrio, esconde todo el trabajo doméstico no remunerado que ellas realizan, por ello, este ejercicio se dedica a visibilizar de manera aproximada cuánto valor genera el trabajo doméstico no remunerado en relación al trabajo remunerado de sus parejas. Para esto se toma el salario o ingresos recibidos por los esposos mensualmente $(\mathrm{sBU})^{8}$ como el monto base para este ejercicio de medición económica (ver Tabla 5).

La presente operación (actualizada para el SBU 2019) requiere como primer punto obtener la sumatoria de las horas semanales de las actividades domésticas seleccionadas, es decir, la sumatoria del tiempo invertido de lunes a domingo, para luego multiplicar por las 4 semanas que componen un mes; por tanto, el total de horas semanales de actividades domésticas de cuidado no remuneradas de las mujeres madres jefas de hogar resultó ser de 122 horas. Por otro lado se 
Tabla 5. Fórmula para visibilizar el valor que el trabajo doméstico genera en relación al SBU

\begin{tabular}{|c|c|}
\hline Fórmula & Operaciones y resultados \\
\hline 1) $\mathrm{HSM}^{*} 4=\mathrm{HMM}$ & $30: 30^{*} 4($ semanas del mes $)=122$ \\
\hline $\mathrm{HMM} * \mathrm{CHSBU}=\mathrm{CMMNR}$ & $122^{*} \$ 1,64($ costo hora SBU $)=\$ 200,08$ \\
\hline 3) $\mathrm{SBU}(\$ 394)+\mathrm{CMMNR}=\mathrm{CDEP}$ & $\$ 394+\$ 200,08=594$ \\
\hline Variables & $\begin{array}{l}\text { (HSM: horas semanales mujer; HMM: } \\
\text { horas mensuales mujer; CHSBU: costo } \\
\text { hora sueldo básico unificado; CMMNR: } \\
\text { costo mensual mujer no remunerado; } \\
\text { SUB: sueldo básico unificado; CDEP: } \\
\text { costo debe empleador pagar) }\end{array}$ \\
\hline
\end{tabular}

Fuente: Encuesta sobre trabajo doméstico y de cuidados (MAU, 2016). Elaboración: propia.

realizó la operación que posibilita obtener el costo por hora de trabajo remunerado del sBU:

USD 394 (SBU)/30 = USD 13,13 (costo por día de trabajo); USD 13,13/8 (horas diarias de trabajo)

$=$ USD 1,64 (costo por hora de trabajo)

De tal forma, la operación de multiplicación de las 122 horas semanales de actividades domésticas de las mujeres por USD 1,64; estipulado para la hora de trabajo remunerado en base al SBU actual, arroja como resultado el valor de USD 200,08 correspondiente al valor aproximado mensual de estas horas de actividades domésticas realizadas por las mujeres del barrio. Por ende, bajo la orientación feminista en la que se advierte que el sistema patriarcal capitalista del trabajo asalariado esconde el trabajo no remunerado de las mujeres, y en relación al enfoque metodológico de que los empleadores deberían pagar/remunerar el trabajo no remunerado que posibilita la subsistencia de la fuerza de trabajo, al sBu debería sumarse el valor de las actividades domésticas mensuales, es decir, que sumando a los USD 394 (SBU) + USD 200,08 (valor por las horas de actividades domésticas mensuales) da como resultado USD 594,08 que el empleador/a debería remunerar o pagar por las horas que el trabajador vende su fuerza de trabajo y por las horas de estas actividades que realizan las jefas de hogar para el bienestar de la fuerza de trabajo.

Como sabemos, el sistema capitalista de trabajo - representado por los dueños, empresarios y empleadores - no reconoce de manera remunerada todo el trabajo doméstico que realizan las mujeres. Si bien es cierto que este cálculo es aproximado y solo ha tomado en cuenta las actividades seleccionadas de una larga lista de tareas que realizan este grupo de mujeres, se logra demostrar que el sBu de los trabajadores no toma en cuenta las horas de trabajo doméstico y de cuidado no remunerado de las mujeres; trabajo que sostiene la vida y la economía de las ciudades, así lo reflexiona, por ejemplo, María Ángeles Durán, cuando se refiere a todo el trabajo no remunerado de los hogares que sostiene la economía de las ciudades y la vida en las mismas (Durán, 2017). No queda duda que sin este trabajo no fuese posible la producción de plusvalía - ganancia que se apropia el mercado capitalista-.

Es importante indicar que este cálculo y procedimiento económico, no pretende afirmar que es la única alternativa para reconocer el trabajo doméstico de las mujeres madres jefas de hogar, y de las mujeres amas de casa en general. Tampoco se pretende afirmar que es el cálculo más justo para medir el trabajo doméstico invisibilizado; sin embargo, logra de todas formas 
reconocer el valor de todo el trabajo que las mujeres realizan en el hogar y que el sistema patriarcal capitalista se ha encargado de negarlo, omitirlo y abusarlo.

\section{CONCLUSIONES}

En términos de los estudios feministas y de género, los cuales analizan y cuestionan las diversas formas de opresión a las mujeres, el estudio sobre la economía feminista y la economía del cuidado profundizan su enfoque en la opresión económica y cultural hacia las mujeres, las cuales radican sobremanera en cómo el pensamiento económico centrado en el hombre económico generó aristas y postulados económicos, muchos de ellos vigentes hasta la actualidad; así, la invisibilización del aporte económico de las mujeres cuidadoras y trabajadoras domésticas no remuneradas de los hogares es un ejemplo. Por otro lado, este tipo de corrientes de pensamientos contribuye a la reproducción del patriarcado a través de la tradición masculina de preservar la servidumbre femenina.

El sistema económico capitalista ha inaugurado en su base cultural el patriarcado como su principal fundamento, y es sobre esto el cuestionamiento económico feminista que en este artículo se presenta. Los estudios económicos tradicionales han excluido a las mujeres como sujetos agentes de economía, siendo afín a la ideología androcéntrica.

En contraposición a esta lectura androcéntrica de la economía, la teoría feminista económica contribuye a desmontar la ideología del patriarcado económico que ubicó al hombre como el centro de la economía; desde una ruptura epistemológica logra confrontar la supuesta ontología de domesticación de la mujer. La teoría feminista logra ubicar en el centro del debate la cuestión de que las actividades domésticas y de cuidado deben ser valoradas y reconocidas como trabajo, es decir, que las diversas actividades en el hogar no solo reproducen la fuerza de trabajo, sino que generan valor en y para el mercado.

Por lo que el estudio de caso analizado en este texto demuestra cómo las mujeres que sostienen la vida de las familias, primero, han construido una concepción del tiempo distinto determinado por el saber cuidar, asimismo, este estudio de caso posibilitó mostrar el uso del tiempo y la valoración monetaria de estas actividades de manera aproximada, lo que provee de un acercamiento importante para reconocer de manera valorativa, económica el trabajo invisibilizado históricamente de las mujeres. Se logra, por lo tanto, identificar que el tiempo y las energías de las mujeres está siendo utilizado en gran medida a cuidar, a desarrollar actividades relacionadas al sostenimiento de la vida de los familiares, parejas y personas cercanas al núcleo social, lo que implica de manera preocupante la falta de tiempo de las mujeres para la recreación, el ocio y el autocuidado, y la falta de reconocimiento digno de que este trabajo no remunerado aporta a la economía de manera fundamental.

\section{NOTAS}

1 El presente artículo forma parte de un estudio más profundo sobre Economía del cuidado, un estudio de caso, realizado para la obtención del título de magíster por la Universidad Andina Simón Bolívar. 2 El barrio María Augusta Urrutia está ubicado al nororiente de la ciudad de Quito, un barrio constituido y liderado por las mujeres. 
3 Gerda Lerner, historiadora austriaca estudiosa de la historia del patriarcado desde sus orígenes.

4 Se han identificado como madres jefas de hogar en tanto además de sostener la vida de sus familias y comunidad, también aportan económicamente a sus familias.

5 Las actividades de trabajo doméstico y de cuidados de las madres jefas de hogar fueron exploradas a través de una encuesta al universo del barrio ( 63 familias) tomando en cuenta una encuesta separada para los esposos o convivientes de las mujeres.

6 En definitiva, la familia también debe incluirse en este enfoque de desnaturalización de la feminización de lo doméstico, sin embargo, se conoce que las hijas mujeres son quienes también realizan estas actividades, así que por ello se ha decidido realizar esta comparación con los esposos o convivientes quiénes no se corresponsabilizan del cuidado y bienestar familiar.

7 Información recolectada a través de herramientas cualitativas como entrevistas.

8 Es importante recordar que esta operación de valorización monetaria del trabajo doméstico de las mujeres del barrio, se la realiza en relación al sBu de sus esposos o convivientes, el promedio mayoritario de ellos recibe un sueldo básico al mes, no se toma en cuenta por cuestiones de alcance de este estudio, las horas extras u otros beneficios que ellos mencionan en la encuesta recibir, además esas particularidades dependen de las instancias, contratos laborales, o acuerdos de trabajo que no es posible tener el control en esta operación.

\section{REFERENCIAS}

Benería, L. (1984). Reproducción, producción y división sexual del trabajo. Santiago de Chile, Chile: ILPES. Borderías, C. (1999). La feminización de los estudios sobre el trabajo. Barcelona, España: Hojas de Pensamiento.

Carrasco, C. (2003). Mujeres y economía. Nuevas perspectivas para viejos y nuevos problemas. Barcelona, España: Icaria.

Carrasco, C. y Gardiner, J. (1999). El marxismo. En C. Carrasco, Mujeres y economía. Nuevas perspectivas para viejos y nuevos problemas (págs. 70-86). Barcelona, España: Icaria.

Consejo Nacional para la Igualdad de Género (CNIG) e Instituto Nacional de Estadística y Censos (INEC). (2018). Cuenta Satélite 2007-2015. Quito, Ecuador: INEC.

Costa, M. R. (2006). Dinero, perlas y rosas en la reproducción feminista. Tres Cantos, España: Akal.

Durán, M. A. (2017). Ciudades que cuidan. En M. N. Rico y O. Segovia, ¿Quién cuida en las ciudades? (págs. 91-116). Santiago de Chile, Chile: CEPAL-NNUU.

England, P. (1993). The separative self: Androcentric bias in neoclassical asumptions. En J. Nelson and M. Ferber, Beyond Economic Man, (págs. 280-292). Chicago, UsA: University of Chicago.

Federici, S. (2010). Calibán y la bruja. Madrid, España: Traficantes de Sueños.

Federici, S. (2013). Revolución a punto cero trabajo doméstico, reproducción y luchas feministas. Madrid, España: Traficante de Sueños.

Ferber, M. y Nelson, J. (2004). Más allá del hombre económico. Madrid, España: Cátedra.

Marx, K. (1867). El capital. Londres, Reino Unido: Luarna.

Millet, K. (2010). Teoría de la Política Sexual. Madrid, España: Cátedra.

Smith, A. (1976). An impiry in to the nature and causes of the wealth of nations. México DF, México: Fondo de Cultura Económica. 
